\title{
Pigmented epithelioid melanocytoma: A rare tumor
}

\section{Meryem Khalidi, Hasna Kerrouch, Mohammed El Amraoui, Nawfal Hjira, Mohammed Boui}

\author{
Dermatology-Venerology Department at Mohammed V Military Hospital of Instruction, Rabat, Morocco
}

Corresponding author: Meryem Khalidi, MD, E-mail: drkhalidimeryem@gmail.com

\begin{abstract}
Pigmented epithelioid melanocytoma is a poorly understood and rarely reported skin tumor which mimics melanoma and whose diagnosis is based on clinical, histological and immunohistochemical arguments. We report the case of a young 24-year-old patient who consulted for the sudden appearance of a plaque and then a black nodule on the left cheek, gradually increasing in size, painless and slightly infiltrated. Dermoscopy could not decide between a nodular melanoma or a blue nevus given the intensity of the pigmentation. Anatomopathological examination of the piece revealed a pigmented epithelioid melanocytoma or animal-type melanocytoma. The consequences of the complete excision were simple without recurrence after a follow-up of 9 months. It is a borderline affection, its diagnosis and treatment are necessary in order to avoid any unforeseeable malignant evolution.
\end{abstract}

Key words: Pigmented epithelioid melanocytoma; Melanoma; Blue nevus

\section{INTRODUCTION}

Pigmented epithelioid melanocytoma or animal-like melanoma is a rare form melanocytic tumor whose name, origin, the diagnosis, the evolutionary potential are the subject of controversy. In almost a century, only a hundred cases has been reported, of which more than two-thirds in the last five years. it is classified as borderline tumor between melanoma and the blue nevi group. It can appear de novo or on a pre-existing lesion, in particular a nevus. We report a new observation of this entity.

\section{CASE REPORT}

S.H, 24 years old, without a notable medical history, having consulted for a papular pigmented lesion of the face that appeared one year ago and rapidly increased in size. The medical examination found a single bluish nodule of $10 \mathrm{~mm}$ diameter on the left cheek, symmetrical and homogeneous, with regular contours without palpable satellite lymphadenopathy (Figs. 1 and 2). Histology of the excisional piece showed that the nodule consisted of large, homogeneous, highly pigmented epithelioid or spindle-shaped melanocytes (Fig. 3). Immunohistochemistry showed that the histiocytic infiltrate was dispersed in melanocytes. Anti HMB45 did not show a maturation gradient and melanocytes were not stained with Ki67. Thus, the diagnosis of pigmented epithelioid melanocytoma was retained. The scar was resumed with $0.5 \mathrm{~cm}$ of margin. The follow-up is 9 months without recurrence.

\section{DISCUSSION}

Pigmented epithelioid melanocytoma (PEM) is a rare skin tumor classified as borderline melanocyte proliferation [1]. It's also called animal-type melanoma because of its first description in white horses. PEM has been described in patients of all ages, although the majority of cases have been in young adults.

Clinically, It is a nodule or plaque, black or blue with a rough surface, oval with an irregular border, measuring 1 to $2 \mathrm{~cm}$, of ubiquitous site and can be seen at any age, including in children [2]. It is predominantly

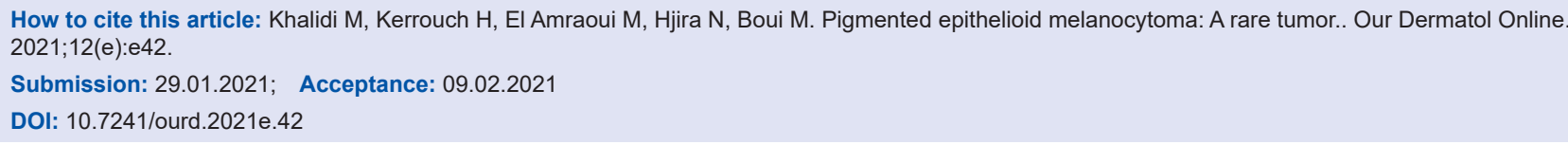




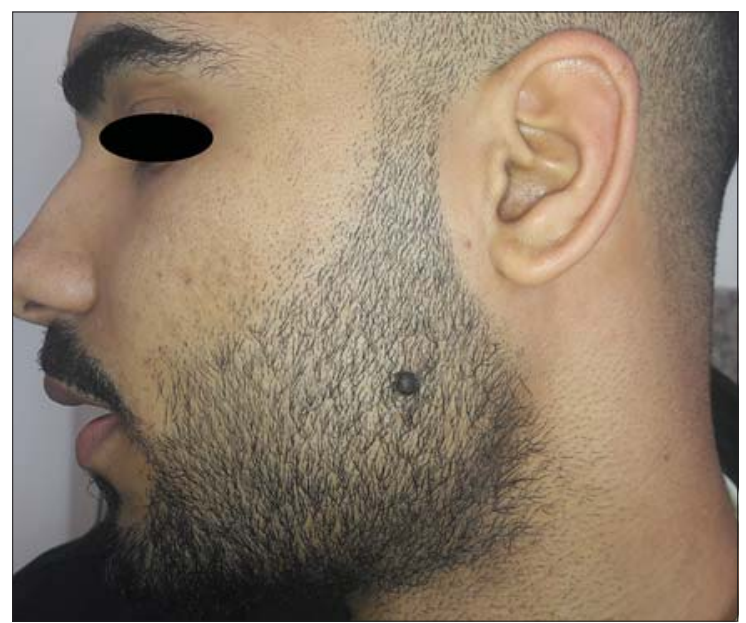

Figure 1: Pigmented epithelioid melanocytoma of the cheek.

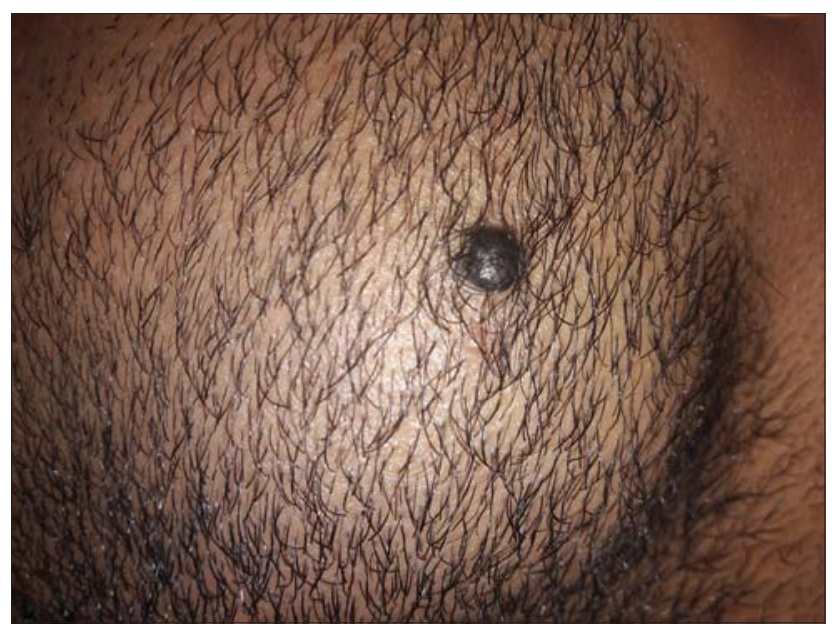

Figure 2: Pigmented epithelioid melanocytoma like a blue nevi.

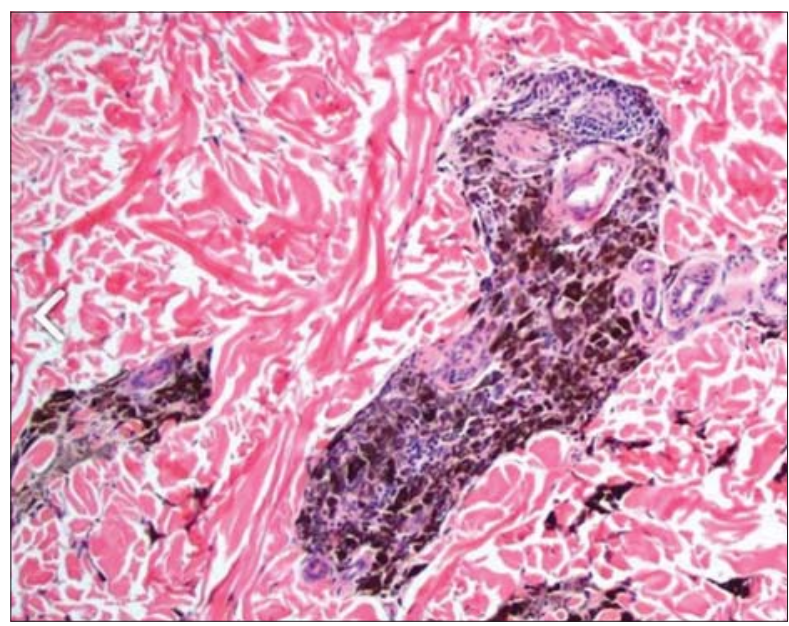

Figure 3: Histopathological pattern of PEM After bleaching, cells have abundant gray slate-like cytoplasm, round nuclei, prominent nuclear membrane and prominent nucleoli.

located on the scalp and the limbs. It generally appears de novo, and no association has been found with prior congenital or acquired melanocytic nevi, although there has been a report of a case occurring in association with a blue nevus. The association between PEM and other predisposing factors, such as a family history of melanoma or sunburn, appears to be anecdotal. The biologic behavior of animal-type melanoma is unpredictable, and both regional lymph node and distant metastases are seen in many cases. Mortality rates, however, are lower than in other types of melanoma [3].

It is defined histologically by the absence of an intraepidermal component and by the presence of a dermal proliferation made of confluent sheets of highly pigmented polygonal cells surrounded by melanophages without inflammatory reaction [4]. Nuclei that are poorly visible due to the intensity of pigmentation have dense chromatin clustered around the periphery and are nucleated. Cytonuclear atypical are rare and mitoses are exceptional. Immunohistochemistry after bleaching of the section with potassium permanganate is necessary to confirm the diagnosis [5].

The histologic differential diagnosis should include other melanocytic lesions such as malignant blue nevus, deep penetrating nevus, melanoma metastasis, certain Spitz nevi, nodular melanoma, and primary dermal melanoma. Histologic diagnosis of animaltype melanoma is difficult, even for pathologists with extensive experience in pigmented tumors.

The dermoscopic features of animal-type melanoma can appear like a homogeneous blue pattern (corresponding to the presence of heavily pigmented melanocytic cells in the dermis), irregular whitish structures (areas of epidermal hyperplasia), and a polymorphous vascular pattern generally composed of large irregular vessels, reflecting the prominent vascular component present in these tumors. These dermoscopic features, however, are not specific to PEM and cannot therefore be used to differentiate between this type of melanoma and any other dermal melanocytic proliferations. Correlation of dermoscopic and histologic findings, however, may be useful in difficult-to-diagnose cases progresses [6].

PEM progress significantly less aggressively than classic melanomas. Their mitotic index is nevertheless low and no regression, ulceration or intra- or peritumoral vascular embolism is generally described (5). Overall, the usual histopronostic criteria are not applicable to this tumor, the progression of which is often indolent, but sometimes more aggressive with local recurrences, lymph node metastases and, more rarely, visceral. 
Enlarged resection and prolonged monitoring are indicated [7].

\section{CONCLUSION}

Pigmented epithelioid melanocytoma is considered as a low-grade melanoma which should be suspected in front of any pigmented lesion and should not be confused with a blue nevus. However its rapid management seems necessary because of the risk of transformation into a real melanoma skin cancer.

\section{Consent}

The examination of the patient was conducted according to the principles of the Declaration of Helsinki.

The authors certify that they have obtained all appropriate patient consent forms, in which the patients gave their consent for images and other clinical information to be included in the journal. The patients understand that their names and initials will not be published and due effort will be made to conceal their identity, but that anonymity cannot be guaranteed.

\section{REFERENCES}

1. Zembowicz A, Carney JA, Mihm MC. Pigmented epithelioid melanocytoma: a low-grade melanocytic tumor with metastatic potential indistinguishable from animal-type melanoma and epithelioid blue nevus. Am J Surg Pathol. 2014;28:31-40.

2. Ward JD, Brady SP, TadaH, Levin NA. Pigmented epithelioid melanocytoma. Int J Dermatol. 2016;45:1403-5.

3. Cecchi R, Rapicano V. Pigment synthesizing (animal type) melanoma with satellite metastasis. Eur J Dermatol. 2017;17:335-6.

4. Sadayasu A, Fujimura T, Haga T, Kambayashi Y, Furudate S, Aiba S. Pigmented epithelioid melanocytoma: immunohistochemical profiles of tumour-infiltrating histiocytes. Acta Derm Venereol. 2013;93:481-2.

5. Mandal RV, Murali R, Lundquist KF, Ragsdale BD, Heenan P, McCarthy SW, et al. Pigmented epithelioid melanocytoma: favorable outcome after 5- year follow-up. Am J Surg Pathol. 2019;33:1778-82

6. Crowson AN, Magro CM, Mihm MC. Malignant melanoma with prominent pigment synthesis: «animal type» melanoma - a clinical and histological study of six cases with a consideration of other melanocytic neoplasms with prominent pigment synthesis. Hum Pathol. 2011;30:543-50.

7. Levene A. Disseminated dermal melanocytosis terminating in melanoma. Br J Dermatol. 2013;101:197-205.

Copyright by Meryem Khalidi, et al. This is an open access article distributed under the terms of the Creative Commons Attribution License, which permits unrestricted use, distribution, and reproduction in any medium, provided the original author and source are credited.

Source of Support: Nil, Conflict of Interest: None declared. 